\title{
Alterations of the default mode network and cognitive impairment in patients with unilateral chronic tinnitus
}

\author{
Yu-Chen Chen ${ }^{1}$, Hong Zhang ${ }^{2}$, Youyong Kong ${ }^{3}$, Han $\mathrm{Lv}^{4}$, Yuexin Cai ${ }^{5}$, Huiyou Chen ${ }^{1}$, Yuan Feng ${ }^{1}$, \\ Xindao Yin ${ }^{1}$ \\ ${ }^{1}$ Department of Radiology, Nanjing First Hospital, Nanjing Medical University, Nanjing 210006, China; ${ }^{2}$ Department of Radiology, The Affiliated \\ Jiangning Hospital of Nanjing Medical University, Nanjing 211100, China; ${ }^{3}$ School of Computer Science and Engineering, Southeast University, \\ Nanjing 210018, China; ${ }^{4}$ Department of Radiology, Beijing Friendship Hospital, Capital Medical University, Beijing 100071, China; ${ }^{5}$ Department of \\ Otolaryngology, Sun Yat-sen Memorial Hospital, Sun Yat-sen University, Guangzhou 510275, China
}

Correspondence to: Hong Zhang. Department of Radiology, The Affiliated Jiangning Hospital of Nanjing Medical University, No.168 Gushan Road, Nanjing 211100, China. Email: yayiba2063@163.com; Xindao Yin, MD, PhD. Department of Radiology, Nanjing First Hospital, Nanjing Medical University, No.68, Changle Road, Nanjing 210006, China. Email: y.163yy@163.com.

Background: Previous studies have demonstrated that cognitive impairment is linked with neurophysiological alterations in chronic tinnitus. This study aimed to investigate the intrinsic functional connectivity (FC) pattern within the default mode network (DMN) and its associations with cognitive impairment in tinnitus patients using a resting-state functional magnetic resonance imaging (rs-fMRI).

Methods: Thirty-five chronic unilateral tinnitus patients, and 50 healthy controls were recruited for rsfMRI scanning. Both groups were age, gender and education level well-matched. The posterior cingulate cortex (PCC) was chosen as the region of interest (ROI) for detecting the FC changes, and determining if these abnormalities were related to a specific cognitive performance and tinnitus characteristic.

Results: Relative to the healthy controls, tinnitus patients showed increased FC between the PCC and the right medial prefrontal cortex (mPFC). Moreover, the enhanced FC between the PCC and right mPFC was correlated with the poorer TMT-B scores $(r=0.474, \mathrm{P}=0.008)$. These correlations were adjusted by age, gender, education level, GM volume, and mean hearing thresholds. The enhanced FC was not correlated with other tinnitus characteristics or cognitive performances.

Conclusions: The enhanced FC pattern of the PCC that is correlated with cognitive impairment in chronic tinnitus patients, especially the executive dysfunction. Enhanced connectivity pattern within the DMN may play a crucial role in neurophysiological mechanism in tinnitus patients with cognitive dysfunction.

Keywords: Chronic tinnitus; functional connectivity (FC); resting-state functional magnetic resonance imaging (rs-fMRI); default mode network (DMN); posterior cingulate cortex (PCC)

Submitted Jun 28, 2018. Accepted for publication Nov 07, 2018.

doi: $10.21037 /$ qims.2018.11.04

View this article at: http://dx.doi.org/10.21037/qims.2018.11.04

\section{Introduction}

Tinnitus is the experience of sound in the ears or head, even with the absence of an external source (1-3). Tinnitus patients often have depression, anxiety, disturbed sleep, and/or concentration difficulties that significantly influence daily life quality (4-8). Besides social and emotional problems, prior research has implicated tinnitus as impacting cognitive domains including executive functions, attention and working memory (9-11). There are only some studies adopting an objective approach to explore the nature of the cognitive impairment, although cognitive 
deficits are prevalent among tinnitus patients. The cognitive dysfunction may be linked with neuropathological changes in chronic tinnitus (11-14). However, the exact neural mechanism of the cognitive impairment which is related to chronic tinnitus remains to be determined.

Resting-state functional magnetic resonance imaging (rs-fMRI) has been applied to explore the brain function network which is based on the blood-oxygenation leveldependent (BOLD) signal (15). Previous studies have used the rs-fMRI technology to investigate several resting-state networks including the auditory network, the executive control network, and the default mode network (DMN), to explore the neurological mechanisms underlying chronic tinnitus (16-18). The DMN is active at rest and suspended during cognitive activity including several important nodes such as the posterior cingulate cortex (PCC)/precuneus, anterior cingulate cortex (ACC), medial prefrontal gyrus (mPFC), and inferior parietal lobule (IPL), which may predict cognitive impairment $(19,20)$. Using rs-fMRI, several studies have observed an enhanced functional connectivity (FC) within the DMN or between the DMN in chronic tinnitus (21-25). Vanneste et al. investigated for the first time that tinnitus patients showed cognitive deficits, which were related to abnormal activity in several brain regions including the hippocampus, anterior cingulate and insula using a resting-state source localized EEG (12). Nevertheless, the association between the alterations of $\mathrm{DMN}$ and cognitive impairment in chronic tinnitus is still far from clear.

As the key region of DMN, the PCC plays a pivotal role in emotion and distressing information processing $(21,26)$. PCC contains diverse cognitive functions such as visuospatial memory, and emotional and non-emotional information processing $(27,28)$. Moreover, the PCC is also responsible for self-referential processing and social cognition (29). During cognitive processing, the PCC is functionally linked to the DMN regions, such as the ACC and mPFC (26). Thus, cognitive deficits in tinnitus patients may be linked with the FC alterations of PCC. Our prior study has detected an enhanced amount of spontaneous neural activity in the PCC of tinnitus patients (30). Compared to the controls, tinnitus patients exhibited significantly enhanced connectivity between the right anterior insula and left PCC. However, the abnormal neural activity of the PCC related to cognitive impairment in tinnitus still remains unclear.

Regarding the pivotal role of the PCC in tinnitus neuropathology, we aimed to employ a seed-based method to explore the FC patterns of the PCC and its associations with the cognitive impairment in chronic tinnitus compared with healthy controls. We hypothesized that abnormal FC patterns of the PCC within the DMN could be observed in tinnitus patients with cognitive impairment and would correlate with cognitive performance deficits.

\section{Methods}

\section{Participants}

A total of 35 right-sided chronic tinnitus patients from the Nanjing First Hospital Department of Otolaryngology and 50 healthy controls through an online advertisement campaign were recruited (aged between 30 and 70 years, all right-handed and with at least 8 years of education). Both groups were age, gender, and education well-matched. Tinnitus distress was evaluated by the Iowa version of the Tinnitus Handicap Questionnaire (THQ) (31). Ten patients had mild tinnitus, 10 had moderate tinnitus, and 15 had severe tinnitus, according to guideline (32). Hearing thresholds were measured by pure tone audiometry (PTA) at the frequencies of $0.25,0.5,1,2,4$, and $8 \mathrm{kHz}$. There were no statistical differences in six frequencies (mean PTA $\leq 25 \mathrm{~dB}$ ) between two groups (Table 1 and Figure 1). The Self-Rating Depression Scale (SDS) and Self-Rating Anxiety Scale (SAS) were used to assess the depression and anxiety status $(33,34)$. Patients with hyperacusis were excluded according to the Hyperacusis Questionnaire (35). Participants were also excluded if they acknowledged having a history of Meniere's diseases, pulsatile tinnitus, ototoxic drug therapy and surgery, hearing aid use, noise exposure, severe smoking, stroke, alcoholism, Alzheimer's disease, Parkinson's disease, epilepsy, traumatic brain injury, and major medical conditions (e.g., cancer, thyroid dysfunction, severe heart diseases, damaged liver or kidney function). This study was approved by the Research Ethics Committee of the Nanjing Medical University. Written informed consent was acquired from all subjects.

\section{Neurocognitive tests}

Objective cognitive assessment, including global cognitive tests and an extensive neuropsychological test battery, were administered to assess the neurocognitive state. Global cognitive tests contained the Mini Mental State Exam (MMSE) (36) and the Montreal Cognitive Assessment (MoCA) (37). The neurocognitive tests included the 
Table 1 Demographics, clinical, and cognitive characteristics of right-sided tinnitus patients and healthy controls

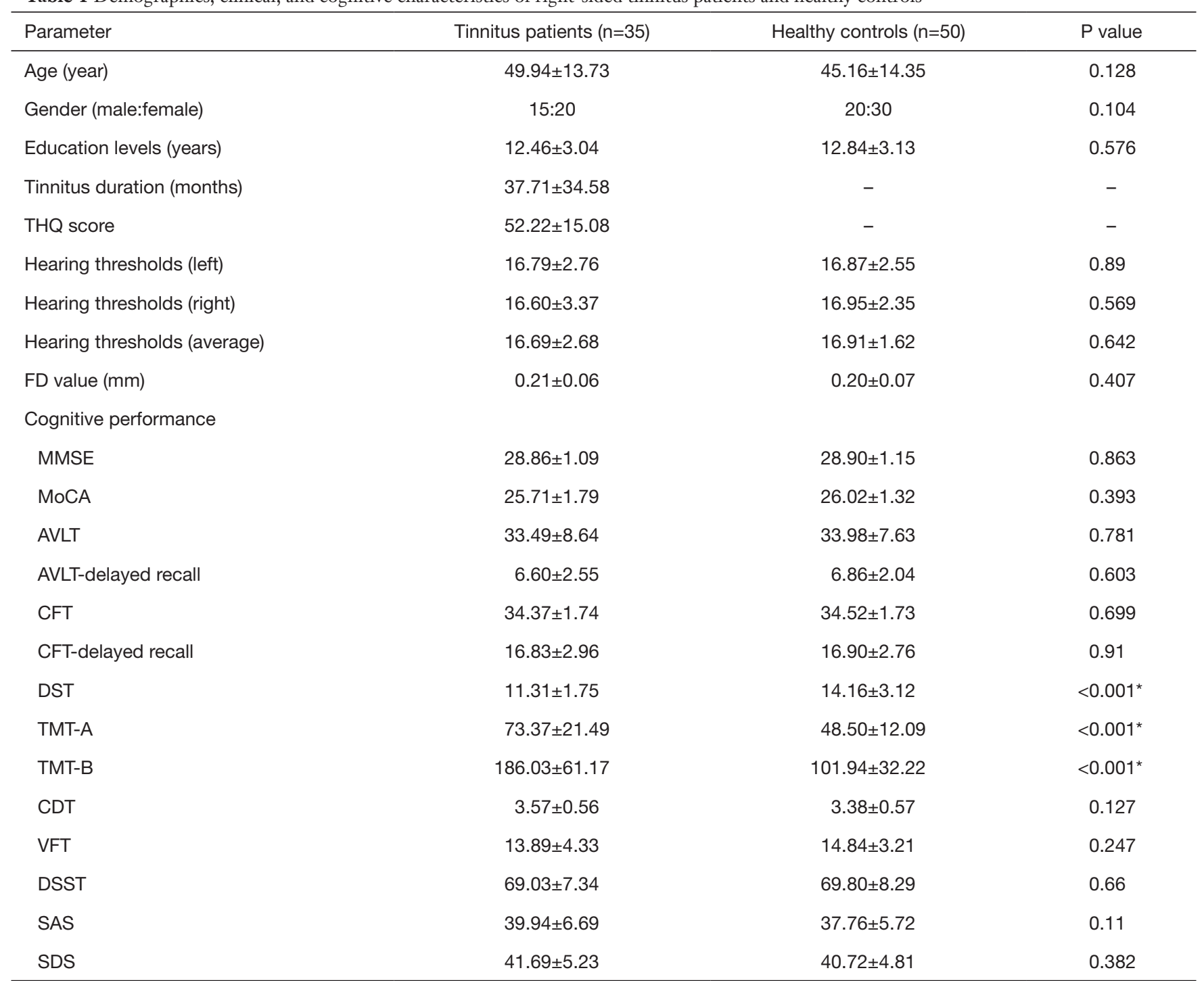

Data are represented as mean \pm SD. The PTA from both ears was averaged. *, P<0.05. PTA, puretone audiometry; FD, framewise displacement; MMSE, Mini Mental State Exam; MoCA, Montreal Cognitive Assessment; AVLT, Auditory Verbal Learning Test; CFT, Complex Figure Test; DST, Digit Span Test. TMT-A, Trail Making Test-Part A; TMT-B, Trail Making Test-Part B; CDT, Clock Drawing Test; VFT, Verbal Fluency Test; DSST, Digit Symbol Substitution Test; SDS, Self-Rating Depression Scale; SAS, Self-Rating Anxiety Scale.

Auditory Verbal Learning Test (AVLT) (38), Rey-Osterrieth Complex Figure Test (CFT) (39), Digit Span Test (DST) (40), Trail Making Test-A and B (TMT-A and TMT-B) (41), Clock Drawing Test (CDT) (42), Verbal Fluency Test (VFT) (43), and Digit Symbol Substitution Test (DSST) (44).

\section{MRI acquisition}

The participants were scanned using a $3.0 \mathrm{~T}$ MRI scanner
(Ingenia, Philips Medical Systems, Netherlands), with an 8-channel receiver array head coil. Subjects lay supine with their head fixed by foam pads and a belt to minimize head motion. And earplugs were used to reduce scanner noise by approximately $32 \mathrm{~dB}$ according to the manufacture's data sheet. The subjects were instructed to lie quietly and keep their eyes closed but not to fall asleep, not to think of anything special, and to avoid head motion during the functional MRI. Structural images were obtained using a three-dimensional turbo fast echo T1WI sequence using 


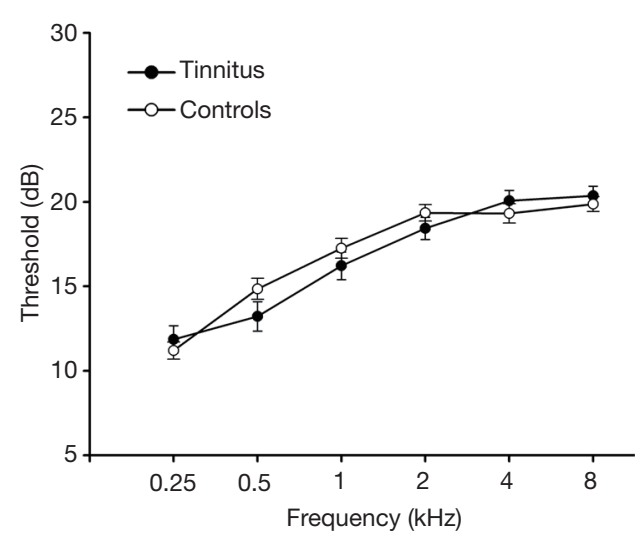

Figure 1 No significant differences in auditory thresholds between chronic tinnitus patients and healthy controls. Data are presented as Mean \pm SEM. SEM, standard error of mean.

the following parameters: repetition time (TR)/echo time $(\mathrm{TE})=8.1 / 3.7 \mathrm{~ms}$; slices $=170$; thickness $=1 \mathrm{~mm}$; gap $=0 \mathrm{~mm}$; flip angle $(\mathrm{FA})=8^{\circ}$; acquisition matrix $=256 \times 256$; field of view $(\mathrm{FOV})=256 \times 256 \mathrm{~mm}^{2}$. The functional images were obtained axially using the gradient echo-planar imaging sequence as follows: $\mathrm{TR} / \mathrm{TE}=2,000 / 30 \mathrm{~ms}$; slices $=36$; thickness $=4 \mathrm{~mm}$; gap $=0 \mathrm{~mm} ; \mathrm{FOV}=240 \times 240 \mathrm{~mm}^{2}$; acquisition matrix $=64 \times 64 ; \mathrm{FA}=90^{\circ}$; and voxel size $=3.75 \times 3.75 \times 4.0 \mathrm{~mm}^{3}$. The SENSE is used for parallel imaging.

\section{Data preprocessing}

fMRI data was preprocessed using Data Processing \& Analysis for (Resting-State) Brain Imaging (DPABI_ V2.3_170105) (45), with the following stages. The first 10 volumes were discarded, followed by a slice-timing adjustment, realignment for head-motion correction, and spatial normalization to the Montreal Neurological Institute (MNI) template (resampling voxel size $=3 \times 3 \times 3 \mathrm{~mm}^{3}$ ) in addition to smoothing with an isotropic Gaussian kernel (FWHM $=6 \mathrm{~mm}$ ), detrending and filtering $(0.01-0.08 \mathrm{~Hz}$ ). Participants with a head motion of $>2.0 \mathrm{~mm}$ translation, or a $2.0^{\circ}$ rotation in any direction, were excluded to minimize movement artifacts.

\section{Structural MRI analysis}

Structural MRI data were processed based on the VBM toolbox (http://dbm.neuro.uni-jena.de/vbm). Briefly, cerebral tissues were segmented into gray matter (GM), white matter (WM), and cerebrospinal fluid (CSF) categories using the unified segmentation model (46). Brain parenchyma volume was measured as the sum of the GM and WM volumes. The GM, WM, and brain parenchyma volumes were divided by the total intracranial volumes, to correct for variability of head size. GM intensity maps in the MNI space were acquired by the unified segmentation algorithm. Data were spatially smoothed with $8 \mathrm{~mm}$ full width at half maximum Gaussian kernel.

\section{Functional MRI analysis}

The region of interest (ROI) of the PCC was developed from a Brodmann template, using the WFU_PickAtlas Version 3.0.5 software (http://fmri.wfubmc.edu/software/ PickAtlas) (47). Briefly, the mean time series of the PCC was obtained for use as a reference time course. Then, Pearson's correlation coefficients were calculated between the average signal change in the ROI (PCC) and the time series of each voxel. Finally, correlation coefficients were converted to $z$ values using Fisher's z-transformation to standardize the statistical analysis (48). Six head motion parameters and a mean time series of global, WM and CSF signals were included as confounding factors in the regression analysis to remove their possible effects.

Between-group analyses were conducted to analyze FC differences between the tinnitus patients and the healthy controls using a whole-brain mask. Age, gender, education level, GM volume, and average hearing thresholds were added as the nuisance covariates. Multiple comparison corrections were performed using a threshold $(\mathrm{P}<0.01)$ of the individual voxel and a cluster size, based on the Monte Carlo simulations (49), corresponding to cluster-level $\mathrm{P}<0.01$ by AlphaSim correction.

\section{Statistical analysis}

Independent $t$-tests and $\chi^{2}$-tests were calculated to investigate the differences in the demographic variables, and cognitive performance scores between tinnitus patients and the controls. Briefly, the mean Z-values of each brain region that showed significant differences were extracted within each subject. Then we performed Pearson's correlation analyses between the mean $\mathrm{Z}$-values and each variable using SPSS (SPSS 19.0, Inc., Chicago, IL, USA). Partial correlations were analyzed using age, gender, education level, GM volume, and average hearing thresholds as covariates. $\mathrm{P}<0.05$ was considered to indicate a statistically 
Table 2 Comparisons of the brain volumes between right-sided tinnitus patients and healthy controls

\begin{tabular}{|c|c|c|c|}
\hline Brain volume & Tinnitus patients $(n=35)$ & Healthy controls $(n=50)$ & $P$ value \\
\hline White matter volume (\% of TIV) & $29.8 \pm 1.5$ & $29.5 \pm 1.6$ & 0.479 \\
\hline Brain parenchyma volume (\% of TIV) & $61.6 \pm 3.0$ & $61.9 \pm 3.2$ & 0.618 \\
\hline
\end{tabular}

Data are expressed as mean $\pm \mathrm{SD}$. TIV, total intracranial volume.
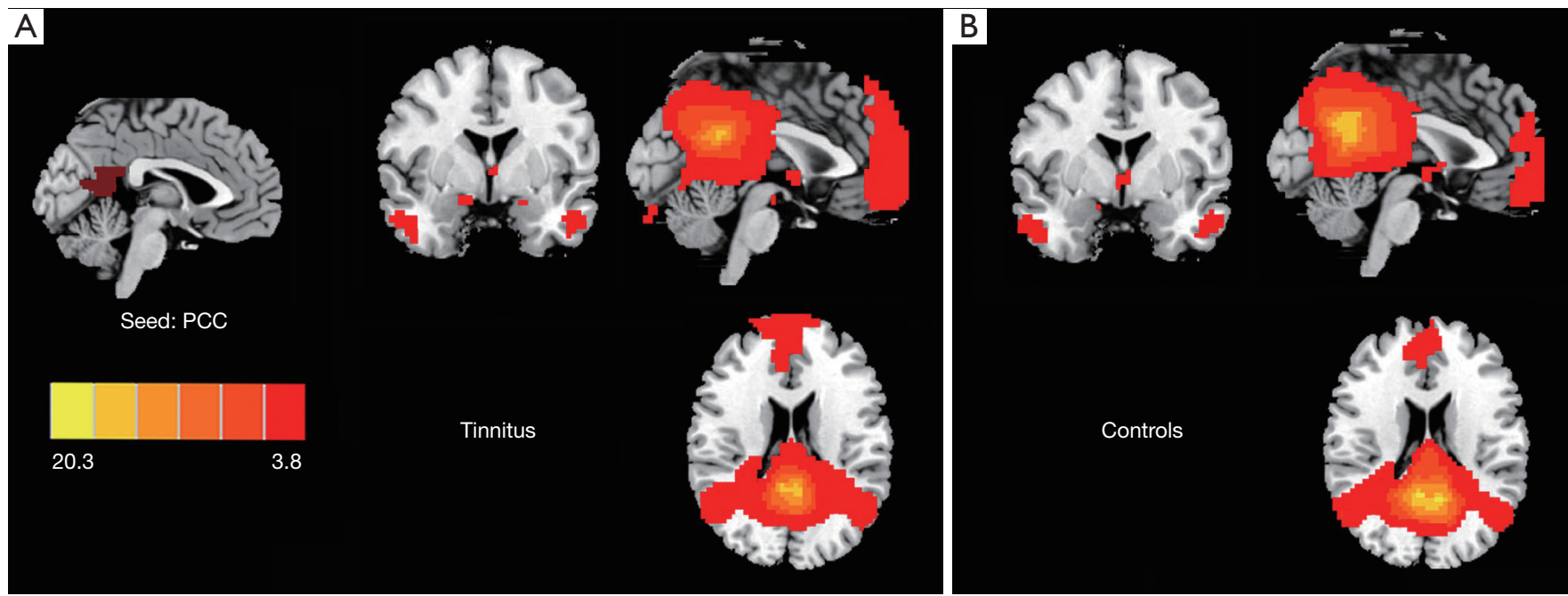

Figure 2 Significant FC patterns of the PCC in whole brain using one-sample $t$-test in chronic tinnitus patients (A) and healthy controls (B). Significant thresholds were corrected using AlphaSim criterion and set at $\mathrm{P}<0.01$. FC, functional connectivity; PCC, posterior cingulate cortex.

significant difference.

Due to the effects of micro-movements from volume to volume on the FC (50), framewise displacement (FD) was calculated for every individual to represent the temporal derivative of the movement parameters. There were no significant differences of the FD values between chronic tinnitus and healthy controls. No participants had FD $>0.5 \mathrm{~mm}$ on more than 35 volumes (Table 1).

\section{Results}

\section{Demographic and neurocognitive data}

The demographic and characteristic data of tinnitus patients and healthy controls were showed in Table 1. The two groups did not differ significantly in age, gender, education level, and average hearing thresholds. Relative to the healthy controls, tinnitus patients were revealed to have a significantly worse performance on the DST, TMT-A and TMT-B tests $(\mathrm{P}<0.05)$. However, the other neuropsychological tests showed slight but no significant decreases in cognitive performance $(\mathrm{P}>0.05)$.

\section{Structural MRI}

No significant differences of GM and WM volumes between tinnitus patients and healthy controls were observed (Table 2). Moreover, no suprathreshold voxel-wise differences of the GM and WM volume between tinnitus patients and controls were demonstrated after Monte Carlo correction.

\section{Functional MRI}

The PCC exhibited strong FC to several DMN regions, including the medial prefrontal cortex (mPFC), IPL, and precuneus in both tinnitus patients (Figure $2 A$ ) and healthy controls (Figure 2B). Compared with the healthy controls, chronic tinnitus patients showed a significantly increased FC between the PCC and the right mPFC (Figure $3 A$ and Table 3). 


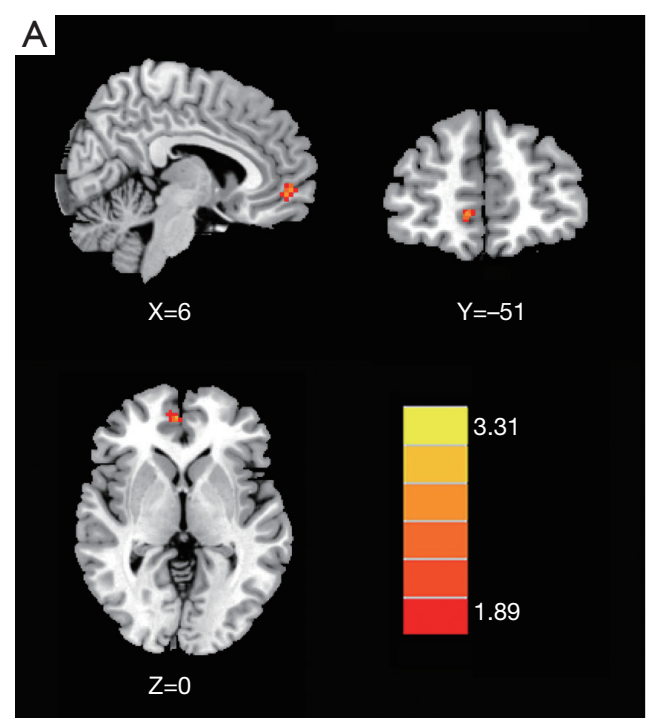

\section{B}

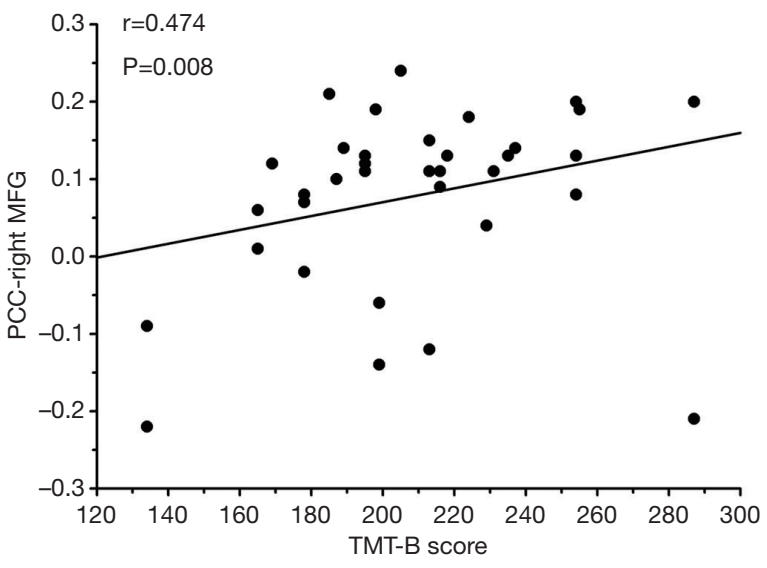

Figure 3 Significant correlations between enhanced FC and poorer cognitive performance. (A) Compared with the healthy controls, chronic tinnitus patients exhibited enhanced FC between the PCC and the right MFG ( $\mathrm{P}<0.01$ corrected by AlphaSim); (B) positive correlations between enhanced FC between the PCC and right MFG and poorer TMT-B scores in tinnitus patients ( $\mathrm{r}=0.474, \mathrm{P}=0.008)$. FC, functional connectivity; PCC, posterior cingulate cortex; MFG, middle frontal gyrus.

Table 3 Increased functional connectivity of PCC in right-sided tinnitus patients compared to healthy controls

\begin{tabular}{llccc}
\hline Brain region & BA & MNI coordinates, $x, y, z(m m)$ & T score & Voxels \\
\hline$R$ medial prefrontal cortex & 10 & $6,-51,0$ & 3.2819 & 52 \\
\hline
\end{tabular}

The threshold was set at a $\mathrm{P}<0.01$ (AlphaSim corrected). BA, Brodmann's area; MNI, Montreal Neurological Institute; PCC, posterior cingulate cortex; $\mathrm{R}$, right.

\section{Correlation analysis}

After correcting for age, gender, education, GM volume, and mean hearing thresholds, chronic tinnitus patients showed enhanced FC of the PCC to the right mPFC, which was positively correlated with the poorer TMT-B scores $(\mathrm{r}=0.474, \mathrm{P}=0.008)$ (Figure $3 B)$. None of the enhanced FC were correlated with other tinnitus characteristics, or cognitive performances.

\section{Discussion}

In this study, the seed-based approach was used to detect the intrinsic FC of the PCC, and its associations with the cognitive impairment in unilateral chronic tinnitus patients. Relative to the controls, chronic tinnitus patients were revealed to have a significantly increased FC between the PCC and the right mPFC, which was positively and specifically linked with the poorer TMT-B scores.
We performed multidimensional neurocognitive tests to assess the cognitive function of every subject in this study. However, except for the DST, TMT-A and TMT-B, most tests exhibited insignificant differences between groups. The DST and TMT scores in tinnitus, exhibited significant reducing trends when compared to the scores in the control group, suggesting that long-term tinnitus perception may lead to overt damage to memory and executive function in tinnitus patients.

No structural changes of GM and WM volumes were detected between tinnitus patients and healthy controls. Prior studies have reported decreases or increases in GM volume in tinnitus patients compared with healthy controls in several brain regions (51-55). However, this was in accordance with our previous studies (30), which could probably be due to the absence of any hearing loss up to $8 \mathrm{kHz}$ in normal hearing tinnitus patients. Moreover, the MRI analyzing technique may result in the discrepancy, 
which was not sensitive enough to detect any differences in GM volume of the patients. Nonetheless, the current results imply that aberrant FC within the DMN related to tinnitus may occur prior to any major structural abnormalities.

DMN disruptions have been previously documented in chronic tinnitus patients $(16,56,57)$. However, no current study has discussed the association between the DMN abnormalities, and cognitive impairment. Our study showed a decreased FC within the DMN was associated with poorer cognitive performance in the chronic tinnitus patients, suggesting that the DMN may be vulnerable to chronic tinnitus with mild cognitive impairment at the early stage. Within the DMN, the PCC functions as the central hub, as well as being a metabolically active and highly connected region in the brain (26). This region is responsible for memory, mediation, emotion, and intrinsic control networks. Decreased FC of the PCC may therefore have contributed to the patients' poorer DST performance. Our prior studies have detected aberrant spontaneous brain activity of the PCC in chronic tinnitus (30), but it did not demonstrate the relationship with the cognitive dysfunction due to tinnitus. Anyhow, this neurocognitive relationship probably supports the hypothesis that an abnormal FC pattern of the PCC may play an important role in tinnitusrelated cognitive dysfunction.

Furthermore, the prefrontal cortex is involved in executive function and emotional processing (58). Previous fMRI studies have detected an enhanced connectivity of the mPFC in chronic tinnitus patients (53,59-61). Similarly, when compared to healthy controls, our tinnitus patients exhibited increased PCC connectivity to the mPFC, which was correlated with a poorer TMT-B performance. TMT-B score is a neurocognitive test that reflects the function of the prefrontal cortex, and it has been commonly used to define cognitive impairments, especially the executive dysfunction (62). Moreover, the mPFC is considered as an important brain region that integrates both the emotional and sensory aspects for tinnitus (63). Araneda et al. suggested that executive dysfunction caused by mPFC abnormalities may play a key role in the generation and maintenance of chronic tinnitus (60). Besides, our prior study detected higher $\mathrm{FC}$ within the attentional control network involving the mPFC (30). Therefore, enhanced FC between the PCC and the mPFC may imply that the mPFC serves as a main hub within specific functional network affected by tinnitus perception.

This study has several limitations. First, we acknowledge that it is impossible to make causal relations between the enhanced FC, and the cognitive impairment in unilateral chronic tinnitus patients. This is because of the crosssectional design, and the smaller population size. Thus, further studies involving a larger number of participants will be needed to confirm the present conclusions. Second, no diagnostic criteria was used for assessing cognitive dysfunction for tinnitus, and this lack of specific and objective cognitive evaluation limited the interpretation of our results. Moreover, we only chose the PCC as ROI to explore the FC patterns of DMN in tinnitus. The seed region can be extended to other DMN regions, such as ACC and precuneus. Additionally, more researches are needed to acquire structural changes, such as diffusion tensor imaging (DTI), to investigate the basis of the functional dysconnectivity within the DMN. Furthermore, due to the limited sample size, we did not compare the FC patterns in subgroups according to the classification of their tinnitus severity, which needs to be considered in future work. Finally, the scanner noise cannot be completely reduced, which may influence the resting-state functional networks between tinnitus and controls, especially the attention network, which requires to be considered in future researches.

\section{Conclusions}

To conclude, the current study mainly identified the increased FC patterns within the DMN regions that were associated with specific cognitive dysfunction in unilateral chronic tinnitus patients. These findings will illustrate the possible role of the $\mathrm{DMN}$ in tinnitus that may bring about a better understanding of the neurophysiological mechanisms underlying chronic tinnitus.

\section{Acknowledgements}

Funding: This work was supported by a grant from the National Natural Science Foundation of China (No. 81601477), Youth Medical Talents of Jiangsu Province (No. QNRC2016062), China Postdoctoral Science Foundation (No. 2017M610337), Jiangsu Postdoctoral Science Foundation (No. 1701007A), and Nanjing Outstanding Youth Fund (No. JQX17006).

\section{Footnote}

Conflicts of Interest: The authors have no conflicts of interest to declare. 
Ethical Statement: This study was approved by the Research Ethics Committee of the Nanjing Medical University. Written informed consent was acquired from all subjects.

\section{References}

1. Wegger M, Ovesen T, Larsen DG. Acoustic Coordinated Reset Neuromodulation: A Systematic Review of a Novel Therapy for Tinnitus. Front Neurol 2017;8:36.

2. Møller AR. Tinnitus: presence and future. Prog Brain Res 2007;166:3-16.

3. Bauer CA. Tinnitus. N Engl J Med 2018;378:1224-31.

4. Reynolds P, Gardner D, Lee R. Tinnitus and psychological morbidity: a cross-sectional study to investigate psychological morbidity in tinnitus patients and its relationship with severity of symptoms and illness perceptions. Clin Otolaryngol Allied Sci 2004;29:628-34.

5. Langguth B, Kreuzer PM, Kleinjung T, De Ridder D. Tinnitus: causes and clinical management. Lancet 2013;12:920-30.

6. Zeman F, Koller M, Langguth B, Landgrebe M. Which tinnitus-related aspects are relevant for quality of life and depression: results from a large international multicentre sample. Health Qual Life Outcomes 2014;12:7.

7. Langguth B. A review of tinnitus symptoms beyond 'ringing in the ears': a call to action. Curr Med Res Opin 2011;27:1635-43.

8. Bhatt JM, Bhattacharyya N, Lin HW. Relationships between tinnitus and the prevalence of anxiety and depression. The Laryngoscope 2017;127:466-9.

9. Hallam RS, McKenna L, Shurlock L. Tinnitus impairs cognitive efficiency. Int J Audiol 2004;43:218-26.

10. Rossiter S, Stevens C, Walker G. Tinnitus and its effect on working memory and attention. J Speech Lang Hear Res 2006;49:150-60.

11. Wang Y, Zhang JN, Hu W, Li JJ, Zhou JX, Zhang JP, Shi GF, He P, Li ZW, Li M. The characteristics of cognitive impairment in subjective chronic tinnitus. Brain Behav 2018;8:e00918.

12. Vanneste S, Faber M, Langguth B, De Ridder D. The neural correlates of cognitive dysfunction in phantom sounds. Brain Res 2016;1642:170-9.

13. Araneda R, De Volder AG, Deggouj N, Philippot P, Heeren A, Lacroix E, Decat M, Rombaux P, Renier L. Altered top-down cognitive control and auditory processing in tinnitus: evidences from auditory and visual spatial stroop. Restor Neurol Neurosci 2015;33:67-80.

14. Mannarelli D, Pauletti C, Mancini P, Fioretti A, Greco
A, De Vincentiis M, Fattapposta F. Selective attentional impairment in chronic tinnitus: Evidence from an eventrelated potentials study. Clin Neurophysiol 2017;128:411-7.

15. Fox MD, Raichle ME. Spontaneous fluctuations in brain activity observed with functional magnetic resonance imaging. Nat Rev Neurosci 2007;8:700-11.

16. Husain FT, Schmidt SA. Using resting state functional connectivity to unravel networks of tinnitus. Hear Res 2014;307:153-62.

17. Chen YC, Wang F, Wang J, Bo F, Xia W, Gu JP, Yin X. Resting-State Brain Abnormalities in Chronic Subjective Tinnitus: A Meta-Analysis. Front Hum Neurosci 2017;11:22.

18. Leaver AM, Turesky TK, Seydell-Greenwald A, Morgan S, Kim HJ, Rauschecker JP. Intrinsic network activity in tinnitus investigated using functional MRI. Hum Brain Mapp 2016;37:2717-35.

19. Raichle ME, MacLeod AM, Snyder AZ, Powers WJ, Gusnard DA, Shulman GL. A default mode of brain function. Proc Natl Acad Sci U S A 2001;98:676-82.

20. Buckner RL, Andrews-Hanna JR, Schacter DL. The brain's default network: anatomy, function, and relevance to disease. Ann N Y Acad Sci 2008;1124:1-38.

21. Bush G, Luu P, Posner MI. Cognitive and emotional influences in anterior cingulate cortex. Trends Cogn Sci 2000;4:215-22.

22. Burton $\mathrm{H}$, Wineland A, Bhattacharya M, Nicklaus J, Garcia KS, Piccirillo JF. Altered networks in bothersome tinnitus: a functional connectivity study. BMC Neurosci 2012;13:3.

23. Maudoux A, Lefebvre P, Cabay JE, Demertzi A, Vanhaudenhuyse A, Laureys S, Soddu A. Connectivity graph analysis of the auditory resting state network in tinnitus. Brain Res 2012;1485:10-21.

24. Schmidt SA, Akrofi K, Carpenter-Thompson JR, Husain FT. Default mode, dorsal attention and auditory resting state networks exhibit differential functional connectivity in tinnitus and hearing loss. PloS One 2013;8:e76488.

25. Schmidt SA, Carpenter-Thompson J, Husain FT. Connectivity of precuneus to the default mode and dorsal attention networks: A possible invariant marker of longterm tinnitus. Neuroimage 2017;16:196-204.

26. Fransson P, Marrelec G. The precuneus/posterior cingulate cortex plays a pivotal role in the default mode network: Evidence from a partial correlation network analysis. Neuroimage 2008;42:1178-84.

27. Sestieri C, Shulman GL, Corbetta M. The contribution of the human posterior parietal cortex to episodic memory. 
Nat Rev Neurosci 2017;18:183-92.

28. Leech R, Sharp DJ. The role of the posterior cingulate cortex in cognition and disease. Brain 2014;137:12-32.

29. Ioannidis S, Roseman H, Chan K, Duckett S, Mizoguchi R. Posterior cortical atrophy: the value of neuroimaging in assessing memory loss. Quant Imaging Med Surg 2017;7:736-9.

30. Chen YC, Zhang J, Li XW, Xia W, Feng X, Qian C, Yang XY, Lu CQ, Wang J, Salvi R, Teng GJ. Altered Intra-and Interregional Synchronization in Resting-State Cerebral Networks Associated with Chronic Tinnitus. Neural plasticity 2015;2015:475382.

31. Kuk FK, Tyler RS, Russell D, Jordan H. The psychometric properties of a tinnitus handicap questionnaire. Ear Hear 1990;11:434-45.

32. McCombe A, Baguley D, Coles R, McKenna L, McKinney C, Windle-Taylor P. Guidelines for the grading of tinnitus severity: the results of a working group commissioned by the British Association of Otolaryngologists, Head and Neck Surgeons, 1999. Clin Otolaryngol Allied Sci 2001;26:388-93.

33. Zung WW. Zung Self-Rating Depression Scale and Depression Status Inventory. In: Sartorius N, Ban TA. Editors. Assessment of Depression. Berlin, Heidelberg: Springer, 1986;221-31.

34. Zung $W W$. A rating instrument for anxiety disorders. Psychosomatics 1971;12:371-9.

35. Khalfa S, Dubal S, Veuillet E, Perez-Diaz F, Jouvent R, Collet L. Psychometric normalization of a hyperacusis questionnaire. ORL J Otorhinolaryngol Relat Spec 2002;64:436-42.

36. Galea M, Woodward M. Mini-mental state examination (MMSE). Aust J Physiother 2005;51:198.

37. Nasreddine ZS, Phillips NA, Bédirian V, Charbonneau S, Whitehead V, Collin I, Cummings JL, Chertkow H. The Montreal Cognitive Assessment, MoCA: a brief screening tool for mild cognitive impairment. J Am Geriatr Soc 2005;53:695-9.

38. Schmidt M. Rey auditory verbal learning test: A handbook. Los Angeles, CA: Western Psychological Services, 1996.

39. Shin MS, Park SY, Park SR, Seol SH, Kwon JS. Clinical and empirical applications of the Rey-Osterrieth complex figure test. Nat Protoc 2006;1:892.

40. Hale JB, Hoeppner JA, Fiorello CA. Analyzing digit span components for assessment of attention processes. J Psychoeduc Assess 2002;20:128-43.

41. Bowie CR, Harvey PD. Administration and interpretation of the Trail Making Test. Nat Protoc 2006;1:2277-81.
42. Samton JB, Ferrando SJ, Sanelli P, Karimi S, Raiteri V, Barnhill JW. The clock drawing test: diagnostic, functional, and neuroimaging correlates in older medically ill adults. J Neuropsychiatry Clin Neurosci 2005;17:533-40.

43. Mok EH, Lam LC, Chiu HF. Category verbal fluency test performance in Chinese elderly with Alzheimer's disease. Dement Geriatr Cogn Disord 2004;18:120-4.

44. Bettcher BM, Libon DJ, Kaplan E, Swenson R, Penney DL. Digit symbol substitution test. In: Kreutzer JS, DeLuca J, Caplan B. Editor. Encyclopedia of Clinical Neuropsychology. London, NY: Springer, 2011;849-53.

45. Yan CG, Wang XD, Zuo XN, Zang YF. DPABI: data processing \& analysis for (resting-state) brain imaging. Neuroinformatics 2016;14:339-51.

46. Ashburner J, Friston KJ. Unified segmentation. Neuroimage 2005;26:839-51.

47. Maldjian JA, Laurienti PJ, Kraft RA, Burdette $\mathrm{JH}$. An automated method for neuroanatomic and cytoarchitectonic atlas-based interrogation of fMRI data sets. Neuroimage 2003;19:1233-9.

48. Lowe MJ, Mock BJ, Sorenson JA. Functional connectivity in single and multislice echoplanar imaging using restingstate fluctuations. Neuroimage 1998;7:119-32.

49. Ledberg A, Åkerman S, Roland PE. Estimation of the probabilities of $3 \mathrm{D}$ clusters in functional brain images. Neuroimage 1998;8:113-28.

50. Power JD, Barnes KA, Snyder AZ, Schlaggar BL, Petersen SE. Spurious but systematic correlations in functional connectivity MRI networks arise from subject motion. Neuroimage 2012;59:2142-54.

51. Mühlau M, Rauschecker J, Oestreicher E, Gaser C, Röttinger M, Wohlschläger A, Simon F, Etgen T, Conrad B, Sander D. Structural brain changes in tinnitus. Cereb Cortex 2006;16:1283-8.

52. Landgrebe M, Langguth B, Rosengarth K, Braun S, Koch A, Kleinjung T, May A, de Ridder D, Hajak G. Structural brain changes in tinnitus: grey matter decrease in auditory and non-auditory brain areas. Neuroimage 2009;46:213-8.

53. Leaver AM, Seydell-Greenwald A, Turesky TK, Morgan S, Kim HJ, Rauschecker JP. Cortico-limbic morphology separates tinnitus from tinnitus distress. Front Syst Neurosci 2012;6:21.

54. Boyen K, Langers DR, de Kleine E, van Dijk P. Gray matter in the brain: differences associated with tinnitus and hearing loss. Hear Res 2013;295:67-78.

55. Boyen K, de Kleine E, van Dijk P, Langers DR. Tinnitusrelated dissociation between cortical and subcortical neural activity in humans with mild to moderate sensorineural 
hearing loss. Hear Res 2014;312:48-59.

56. Husain FT. Neural networks of tinnitus in humans: elucidating severity and habituation. Hear Res 2016;334:37-48.

57. Lanting C, Wozniak A, van Dijk P, Langers DR. Tinnitusand Task-Related Differences in Resting-State Networks. Adv Exp Med Biol 2016;894:175-87.

58. Alvarez JA, Emory E. Executive function and the frontal lobes: a meta-analytic review. Neuropsychol Rev 2006;16:17-42.

59. Rauschecker JP, Leaver AM, Muhlau M. Tuning out the noise: limbic-auditory interactions in tinnitus. Neuron 2010;66:819-26.

60. Araneda R, Renier L, Dricot L, Decat M, EbnerKarestinos D, Deggouj N, De Volder AG. A key role

Cite this article as: Chen YC, Zhang H, Kong Y, Lv H, Cai Y, Chen H, Feng Y, Yin X. Alterations of the default mode network and cognitive impairment in patients with unilateral chronic tinnitus. Quant Imaging Med Surg 2018;8(10):1020-1029. doi: 10.21037/qims.2018.11.04 of the prefrontal cortex in the maintenance of chronic tinnitus: An fMRI study using a Stroop task. Neuroimage Clin 2017;17:325-34.

61. Cavalcanti K, Brasil-Neto JP, Allam N, Boechat-Barros R. A double-blind, placebo-controlled study of the effects of daily $\mathrm{tDCS}$ sessions targeting the dorsolateral prefrontal cortex on tinnitus handicap inventory and visual analog scale scores. Brain Stimul 2015;8:978-80.

62. Ardila A, Pineda D, Rosselli M. Correlation between intelligence test scores and executive function measures. Arch Clin Neuropsychol 2000;15:31-6.

63. Jastreboff PJ. Phantom auditory perception (tinnitus): mechanisms of generation and perception. Neurosci Res 1990;8:221-54. 